\title{
GLUV: A BALLOON-BORNE HIGH CADENCE ULTRAVIOLET MONITORING TELESCOPE FOR SUPERNOVA SHOCK BREAKOUTS AND EXOPLANET ATMOSPHERES
}

\author{
Rob Sharp*a , B. Tucker ${ }^{\mathrm{a}}$, R. Ridden-Harper ${ }^{\mathrm{a}}$, G. Bloxham ${ }^{\mathrm{a}}$, M. Petkovic ${ }^{\mathrm{a}}$ \\ ${ }^{a}$ Research School of Astronomy \& Astrophysics, The Australian National University, Cotter Road, \\ Weston Creek, ACT 2611, Australia
}

\begin{abstract}
Routine photometric monitoring at near-ultraviolet wavelengths $(<400 \mathrm{~nm})$ is compromised from the ground due to highly variable atmospheric transmission and cloud cover. The GLUV project will mount a modest sized telescope (200 $\mathrm{mm}$ primary) on a series of long-duration high-altitude balloon flights. The wide field camera $\left(\sim 7 \mathrm{deg}^{2}\right)$ will perform high cadence (10-300 second rolling integrations) each night for campaign durations of three to six months. The principle science mission is the early-time detection of supernova shock-breakout at near-ultraviolet wavelengths. Additionally, early design analysis has shown the system is also able to probe the atmospheric composition of exoplanet atmospheres through the combination of UV transit measurements with ground-based measurements at longer wavelengths. In this presentation we consider the specifications for a long-duration balloon platform for such a mission, focusing on the necessary mission requirements (sensitivity, sky coverage, cadence etc.) and the available platform suitability. Particular attention is payed to platform flight altitude and atmospheric transmission.
\end{abstract}

Keywords: supernovae, planets: atmospheres, ultraviolet, balloons,

\section{INTRODUCTION}

Potential access to a new generation of high-altitude $(>10 \mathrm{~km}$ ), long-duration (flight duration $\geq$ month) balloon platforms to which modest astronomical observing facilities might be added raises some exciting opportunities for a new generation of mid-scale observational programs (i.e., developed and operated as a University research program as opposed to a national facility). Contact with one such long-duration high-altitude balloon operator has led our team to propose the GLUV program. Long duration balloon flights offer an unusual set of operational capabilities and challenges. Challenges include:

- Experiments must be small to remain lightweight. This limits sensitivity and diffraction limited image quality.

- Inexpensive, as most science cases we have identified are of fundamental importance but remain somewhat niche.

Advantages include:

- Long periods of uninterrupted observation free from interruptions due to cloud cover etc.

- Access to wavelength ranges difficult or impossible to access from ground based facilities.

After some consideration we have identified a number of frontier science program for which a balloon-based wide-field near-ultraviolet survey telescope will allow important new insights.

\section{SCIENCE DRIVERS}

\subsection{Supernova shock breakout}

Our proposed system can addresses a fundamental concern in our understanding of supernova explosions and their use as standard candles for cosmology. Specifically, this system will measure the shock breakout for supernova explosions. The strength of the shock breakout is directly proportional to the size of the precursor star. The mass and properties of the star

\footnotetext{
*Rob.Sharp@anu.edu.au; +61 (0)2 6125 8035; rsaa.anu.edu.au
} 
will determine how much energy is released in their explosion, thus dictating how bright they appear, thus calibrating our standard candle for cosmology. No current or planned experiment will be able to address this calibration concept due to the unique combination of wavelength coverage (ultraviolet), cadence, and sky coverage.

Type Ia supernova (SN Ia) play a crucial role in our understanding of the Universe. The empirical relation of the light curve size and brightness of supernova enables SN Ia's to be used as precision tools for probing the distance Universe. This technique has led to the discovery of an accelerating expansion due to dark energy, the subject of the 2011 Nobel Prize in Physics. Dedicated surveys have discovered thousands of these explosions, showing dark energy is indistinguishable from a cosmological constant, Einstein's original theory of the Universe, to about $10 \%$. Increasing the precision of the measurements is no longer related to just observing more distant supernovae - experiments are now dominated by systematic errors, rather than statistical errors. The largest of these errors is not knowing the exact brightness of the explosion, as we lack a firm knowledge of their progenitor system, compromising their use as distance indicators.

Traditional supernova surveys only observe objects a few times during their explosion. These supernova searches operate so as to guarantee that most supernovae are found at or past maximum light. However, there is clearly a tremendous amount of information about the explosion contained in the early phases of the light curve, which is critical to properly measuring dark energy. The best survey to date, the SDSS-II supernova search, repeatedly visited the same patch of sky as often as once every-other night and at the time, provided the best constraints yet on the early light curves of Type Ia explosions.

But even with this planned cadence, the combination of weather and interference from the Moon resulted in an actual average time between visits of more than four days, leaving large gaps in the early rise-time light curve. Other surveys, such as PTF and Pan-STARRS, continue moderate cadence searches. Fortuitously the nearby supernova $2011 \mathrm{fe}$ in M101 was observed less than a day after its explosion and this single object led to important constraints on the size of the progenitor $^{[1]}$. However, more examples are needed and our proposed program provides a systematic way to identify them. Using the high-cadence available with the Kepler space telescope (which operates only at red wavelengths), and with just 3 SN Ia found, we have been able to deliver the best measurements of the early-phases of a light-curve and discovered some new physics ${ }^{[2]}$.

Type Ia supernovae are believed to be the result of accretion from a normal star, like what our Sun is now, on to a white dwarf, a very old star like what our Sun will be in billions of years. If this scenario is true, then there should be some observational evidence of the "other" star. Detailed calculations ${ }^{[3]}$ of the collision between the material from the supernova explosion and the donating star and predicts that shock emission will be visible, but the amplitude depends on the size of the secondary (other star), the separation between the two stars, and the viewing angle. A survey that could regularly detect (or definitively not detect) the presence of the shock would deliver tight constraints to the supernova model and determine the progenitor systems of SN Ia. Analysis of 100 well-sampled light curves ${ }^{[4]}$ from the SDSS-II rolling supernova search in an attempt to find evidence for shock emission. They found no sign of this shock, however they could only rule out the largest stars. Simulations show that it is not surprising that no clear shock signature was found, as even with the good planned cadence of SDSS-II, it operated in the red wavelengths were this shock is much weaker and shorter. Likewise, the Kepler survey ruled out most main sequence star companions ${ }^{[2]}$, however as it also operated in the red its sensitivity to shock is low. A high-cadence UV mission is needed for the shock test to be conclusive, and to once and for all solve the debate about the progenitors of SN Ia and help figure dark energy. 


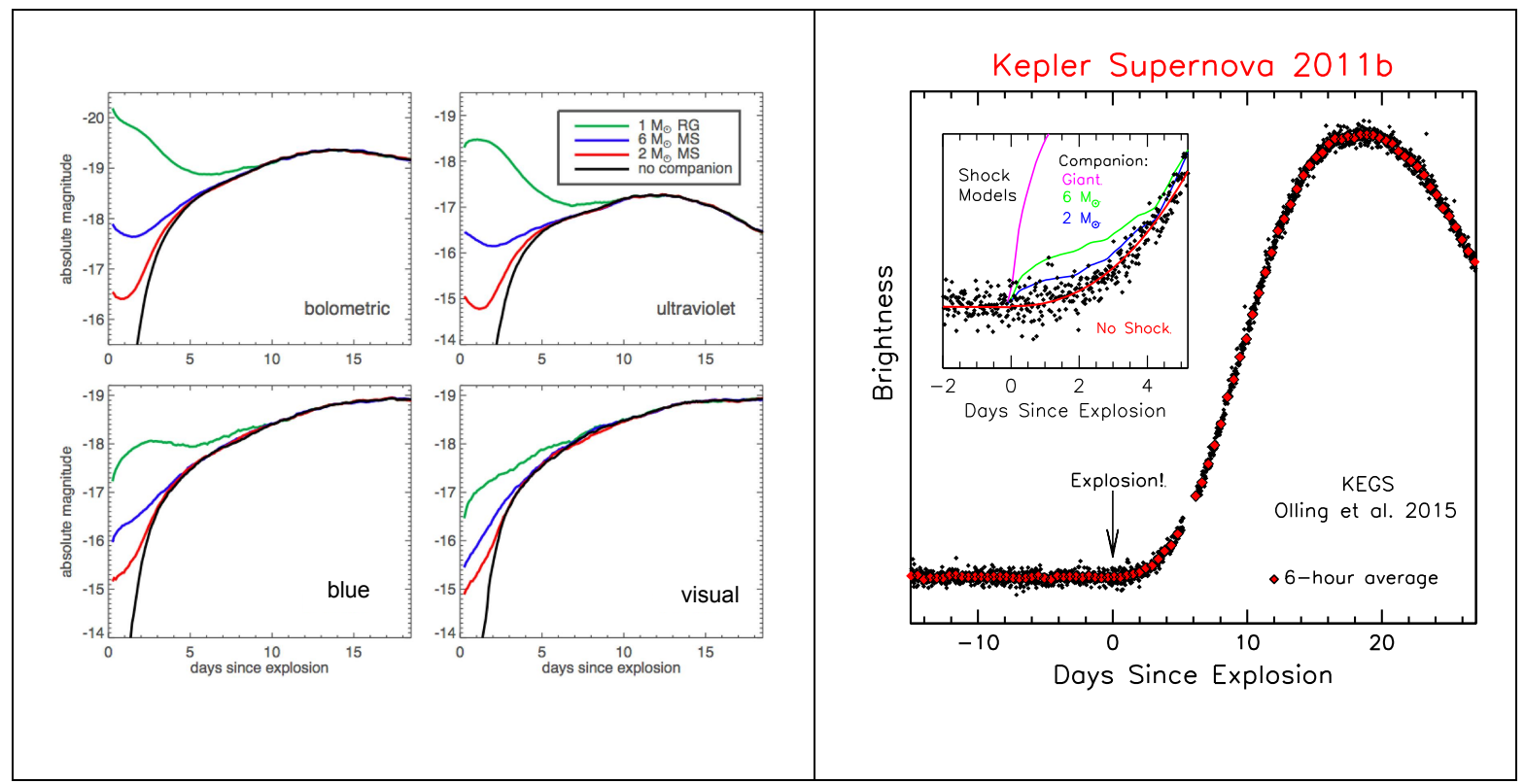

Figure 1: Left - Model light curves of a type Ia supernova from a system involving a white dwarf and another normal star, nominally with a giant star secondary ${ }^{[3]}$. The ejecta shocked by the secondary adds to the supernova light for the first five days or less. The shock is best seen in the ultraviolet, and weak in the optical where most searches operate. With our proposed system, we would easily detect the shock emission more than a factor of 10 fainter than the peak of the

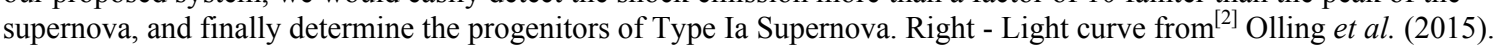
While it can rule out most main sequence star companions, by operating in the optical, it has been shown to be not sensitivity to shock breakout. It is not a matter of just observing this objects early, but in the ultraviolet.

\subsection{Planetary atmospheres during transit}

To find potential habitable planets we need to discover them, determine their location relative to their parent star, and detect an atmosphere. The first two components have become comparatively easy in recent times. The later has proven extremely difficult.

Over the last two decades, there has been a huge international effort to discover planets orbiting other, distant stars, exoplanets. As of last year, there were over 1,000 exoplanets, confirmed, with thousands of candidate planets waiting to be confirmed. The frequency of these objects has now been well determined for exoplanets orbiting their parent stars < 85 days $^{[5]}$. It has also been determined that 1 in 5 Sun-like stars have at least 1 Earth-like planet, meaning there are of order of 20 billion systems like the Sun-Earth in our Milky Way galaxy alone. However, we only know the size of these planets, not their composition.

The focus has now turned to the nature of these distant planets - to understand how planets form, and what are they made of. To understand the nature of a distant planet, we must detected and study its atmosphere, which will determine the place of our own Earth and solar system in a cosmic context, and outline potential environments in our Galaxy for harboring life. However this is extremely difficult through normal optical methods, which are insensitive to planetary atmospheres.

The atmospheres of close-in gas giants (like Jupiter, and Neptune) are strongly dependent on the irradiation they receive. In the near-UV $(200-300 \mathrm{~nm})$, Rayleigh scattering, probes for aerosols and haze produced by photochemistry in the upper atmosphere of these planets. A near-ultraviolet transit mission, working in tandem with transit surveys at longer wavelengths, can use the wavelength dependence of scattering to provide a powerful atmospheric probe. This has been demonstrated in a few cases previously, however it required a combination of the Hubble Space, Spitzer satellite and ground-based facilities. A high-cadence UV telescope that could monitor large areas of sky would be an ideal complement to the array of current ground based transit observatories. 

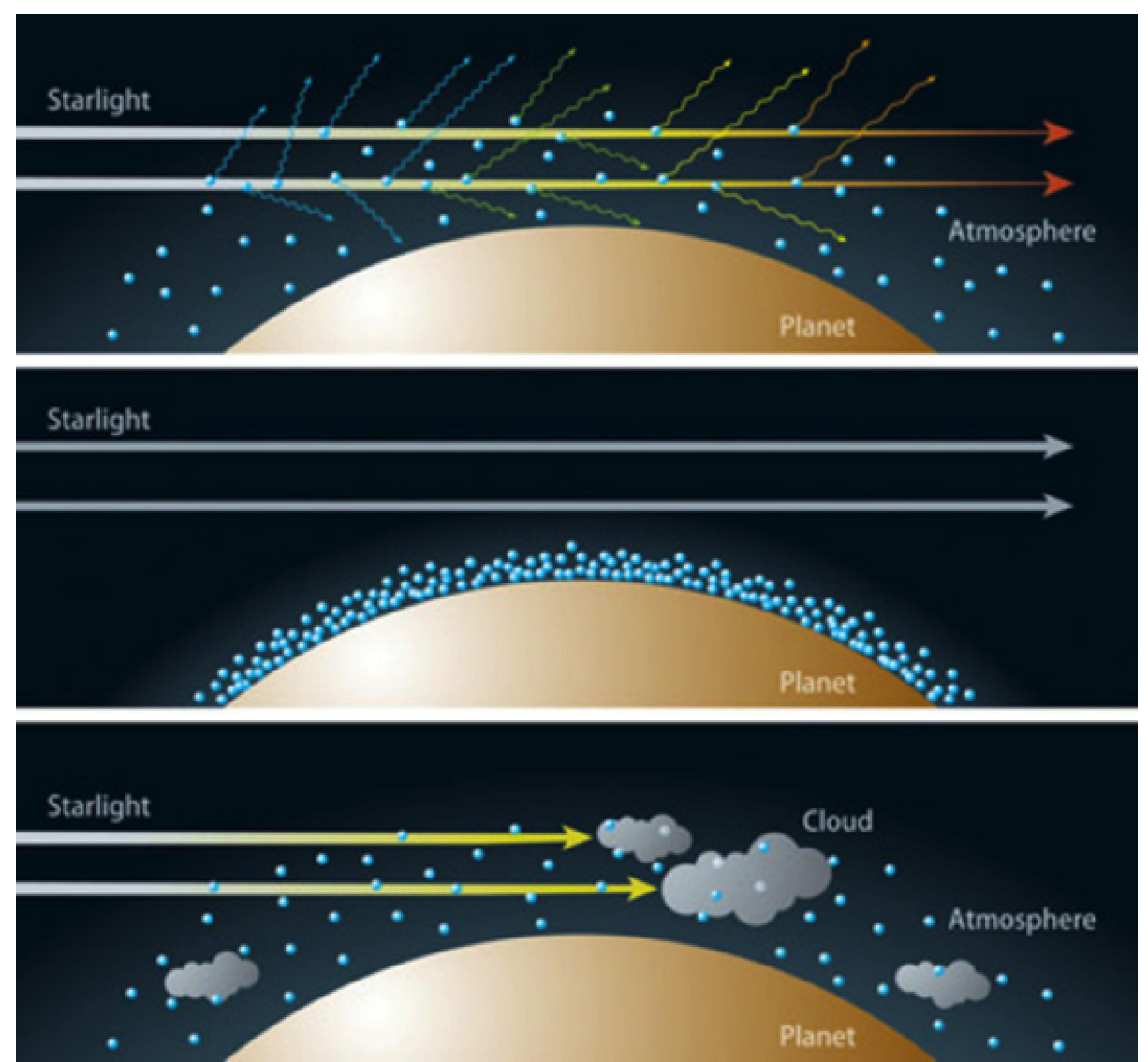

Figure 2: Top - An exoplanet system with an extended hydrogen atmosphere, where blue photons from the parent stars are scattered off, leaving only red photons, which results in a faint UV, but bright red transit. Middle - An exoplanet with a water-dominated atmosphere. In this scenario, the UV photons do not scattering, result in the UV and red transit lightcurves having a similar depth. Bottom - An exoplanet with some water and hydrogen, which causes a variable UV transit.

\subsection{Gravitational wave progenitor systems}

While the detection of GWs produced by coalescing black holes $(\mathrm{BH})$, from the LIGO experiment earlier this year, was revolutionary, it lacked the ability to pinpoint the position of the progenitor system. When LIGO begins its second run later this year, boosted to a greater sensitivity, it is expected to detect 40 NS-NS mergers (Neutron Star pairs), 10 NS-BH mergers and $20 \mathrm{BH}-\mathrm{BH}$ mergers a year, but still without identification of the progenitor system and coordinated follow up observations.

Without knowing the position of the GW source, follow-up multi-messenger observations are not possible, ultimately limiting the interpretation of each event. For this reason a focus is on developing a strong multi-messenger observation network, as it will not only increase the data received from an event but also reduce the GW amplitude detection threshold by a significant factor.

A balloon based high altitude UV telescope with a wide field of view and high cadence would be a valuable facility to pinpoint progenitor systems. The merger events which produce GWs are incredibly energetic, and so often have high luminosity in short wavelengths. Due to the high predicted frequency of NS-NS mergers, it seems reasonable for these events to receive particular attention.

The coalescence of binary neutron star systems to produce a supra-massive NS is discussed by Li \& Yu (2016) ${ }^{[6]}$. A shock breakout is expected to occur after 15 minutes, producing a high soft X-ray luminosity and a small peak in the UV emission with a luminosity $\approx 10^{42} \mathrm{erg} \mathrm{s}^{-1}$. The UV luminosity continues to rise during the mega-nova to a peak value $\approx 10^{44} \mathrm{erg} \mathrm{s}^{-1}$ after 1 day. After 3 days the UV luminosity sharply decreases due to an increasing UV opacity driven by the $r$-process in heavy elements that were ejected during the merger. The GLUV telescope would be an excellent detection 
facility for the early UV signatures of NS-NS coalescences. As the UV luminosity peaks before the optical luminosity, if real-time data is available, the proposed system could initiate multi-messenger follow up observations. The data gathered from such events would provide a valuable insight into the structure of NSs, leading to tighter constrains on the equation of state.

\section{SCIENCE REQUIREMENTS}

Analysis of the science cases outlined above drives design of the proposed UV survey telescope system. While this requirement set is an active area of current development, our baseline estimates at this time are discussed below. Some degree of circularity remains in the requirement definition due to the need to remain within a plausible, but as yet loosely defined mass, and volume and power budget. The short-term goal (2017-2018) of the GLUV program is to demonstrate the viability of a single balloon borne UVB telescope for high cadence survey astronomy. Longer term it is envisaged that a suite of GLUV telescope would be flown (some simple replicas, some with alternative payloads) to achieve greater periods of continuous monitoring coverage and wider survey areas. The science requirements below are designed to deliver a short-term return for a single GLUV unit while demonstrating the potential of the expanded observatory system.

Survey area: To achieve a Supernova rate of in greater than 10 events per year, a survey area of $\geq 20 \mathrm{deg}^{2}$ is required.

This estimate is based on recent SNE rate results from the ongoing Kepler space telescope K2 survey ${ }^{[2]}$, after accounting for gaps due to the daily observational cycle, as well and the local galaxy survey density within the accessible volume for the likely system sensitivity limits.

Instantaneous field of view: To achieve sufficient source monitoring frequency, the instantaneous field of view of the telescope shall be greater than $5 \mathrm{deg}^{2}$.

While survey area can be accrued via multiple independent telescope points, such a strategy limits the period of monitoring of a continuous area of sky (for a single GLUV telescope). It is a premise of the current system design that a continuous tracking telescope mount is not feasible within the project mass and power budget. Instead a limited range tracking system will allow the telescope to monitor dedicated fields for 2 hours a day ( 30 degrees of rotation) allowing 4 fields per assumed dark period of 8 hours duration. Thus a total survey area of $>20 \mathrm{deg}^{2}$ can be monitored at high cadence.

Cadence of observation: To identify transient events, exposure times over a range of duration from 10 seconds to 300 seconds shall be possible with a low readout duty cycle.

Sensitivity and observational cadence are a trade-off for each science program. Planetary transients require high cadence to sample the transit ingress and exit phases. While supernovae detection can tolerate longer dwell times, the critical shock breakout phase is rapid requiring an intermediate exposure time.

Image angular-resolution and sampling: To identify transient events, the instrumental point spread function should be sampled by multiple pixels.

The use of a small telescope on a somewhat unstable platform dictates that image quality, as measured by the effective angular resolution of the recorded images, cannot not be a significant requirement from any realizable system. Therefore, provided one operates significantly above the confusion limit, a large effective image point spread function is of limited concern to the project. Hence a large field of view can be covered with a modest detector format using large pixels. The optics should therefore deliver a PSF that is broadly matched to the expected image residual motion and the camera system should sample this PSF with multiple pixels to ensure a suitable average illumination across pixel boundaries during an exposure.

Sensitivity: To access a sufficiently large survey volume to deliver $>10$ Supernova events a year, a signal-to-noise ratio of $\geq 10$ shall be achieved for an unresolved point source of $m_{U}(A B) \leq 17$ mag for an individual exposure.

The ultimate sensitivity of the proposed survey telescope depends on a number of factors that remain highly uncertain at this time. The two most significant of these are the sky background and atmospheric transmission at the wavelengths of interest (for the likely range of altitudes accessible for operation of the system). These uncertainties will be address in coming months via a detailed analysis of the available atmospheric date along with test flights of a wide-field UV 
radiometer to provide new empirical sky brightness data for comparison with the model expectations at these unusual altitudes.

\section{MISSION PROFILE}

\subsection{Platform availability}

Based on publicly available information, our favored platform for the GLUV mission is that operated as Project Loon (https://www.google.com/loon/). Loon delivers the long duration operations at altitudes relevant near-ultraviolet astronomy. Significant mission profile and design hurdles remain to be addressed to ensure viability of the proposed GLUV mission before it is appropriate to begin formal collaboration. Viability of the GLUV program will be confirmed in the first year of the Preliminary Design Study (2017).

\subsection{Ozone absorption}

A key component to the success of a balloon borne ultraviolet telescope is achieving a flight altitude appreciably above the ozone layer. This requires an understanding of the structure and behavior of the ozone distribution. Not only does the ozone layer density vary with altitude, but it also varies with respect to latitude and season.

The mean density of the ozone layer demonstrates the major latitude variations in ozone density. The ozone density at altitudes below $35 \mathrm{~km}$ features a strong latitudinal variation (Figure 3). Contours of constant ozone density follow a roughly parabolic relationship with latitude, defined by a higher altitude at the equator which decreases towards both poles. The southern hemisphere in general contains less ozone due to the ozone hole created largely by manmade source such as CFCs.

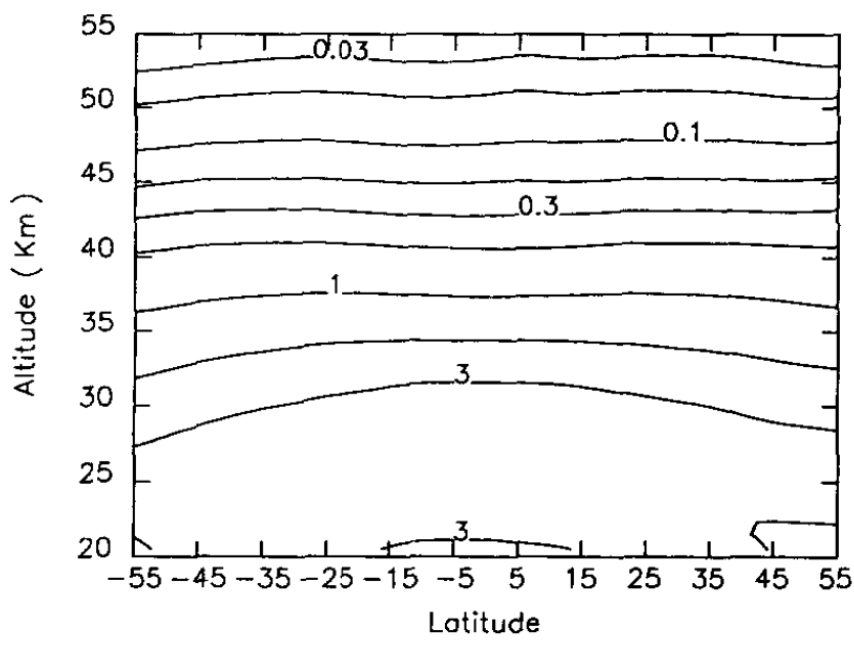

Figure 3: Contours for the yearly mean ozone density $\left(10^{12} \mathrm{~cm}^{-3}\right.$, as measured by the SAGE-I mission) are shown as a function of latitude of observation and altitude. Source: McCormick et al. (1989) ${ }^{[7]}$

The apparent parabolic nature of ozone density contours is produced by Brewer-Dobson Circulation (Figure 4). In this circulation model the production and movement of ozone is driven by the sun; ozone is primarily produced at the equator, due to high solar flux driving the reaction which also drives convection in the stratosphere. Hot air parcels at the equator rise, but they are bounded above by the stratopause and due to mass conservation, the air parcels are forced poleward. As the air parcels move away from the equator they cool and sink to lower altitudes. This process "puffs up" the ozone layer and reduces its overall density at the equator, while compressing the ozone layer to low altitudes and increased density over the mid-latitudes.

The Ozone density also features a strong seasonal variation (Figure 5Figure 5). The two main seasonal variations are the ozone column density and the vertical extent of the ozone layer. As well as acting to create ozone sunlight also breaks down ozone, so in the winter hemisphere ozone column density steadily increase, while in the summer hemisphere the ozone column density decreases, reaching the lowest column density near autumn. Another seasonal variation is the vertical extent of the ozone layer. Due to the higher incident solar radiation during the summer the ozone layer is "puffed 
up" in a similar way as at the equator. The ozone layer in the winter hemisphere is compressed to lower altitudes. Hence the summer hemisphere has a lower column density, but a greater altitude extent, while the winter hemisphere has a higher column density and a lower altitude extent.

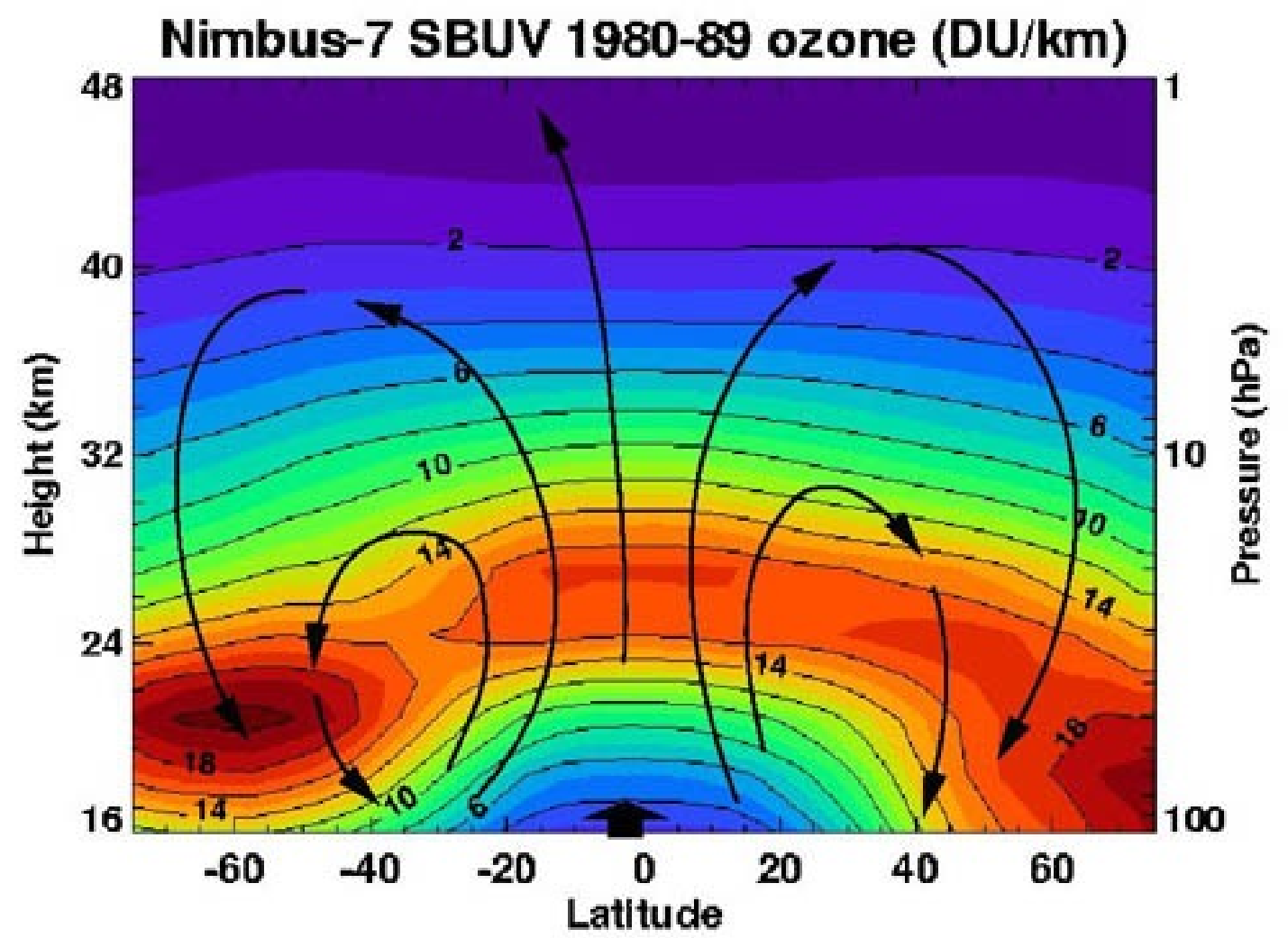

Figure 4: Illustration of the Brewer-Dobson circulation driving latitude variation in atmospheric ozone. Image credit: NASA via Wikimedia Commons.

When considering the structure and variations of the ozone layer a rough flight plan can be constructed. If the flight altitude is greater than $25 \mathrm{~km}$ then it is optimal to fly at latitudes $\mid$ Lat $\mid \geq 30^{\circ}$ in the winter hemisphere, however, if the flight altitude is less than $25 \mathrm{~km}$ it is optimal to fly from the summer hemisphere at $\mid$ Lat $\mid \leq 30^{\circ}$. 


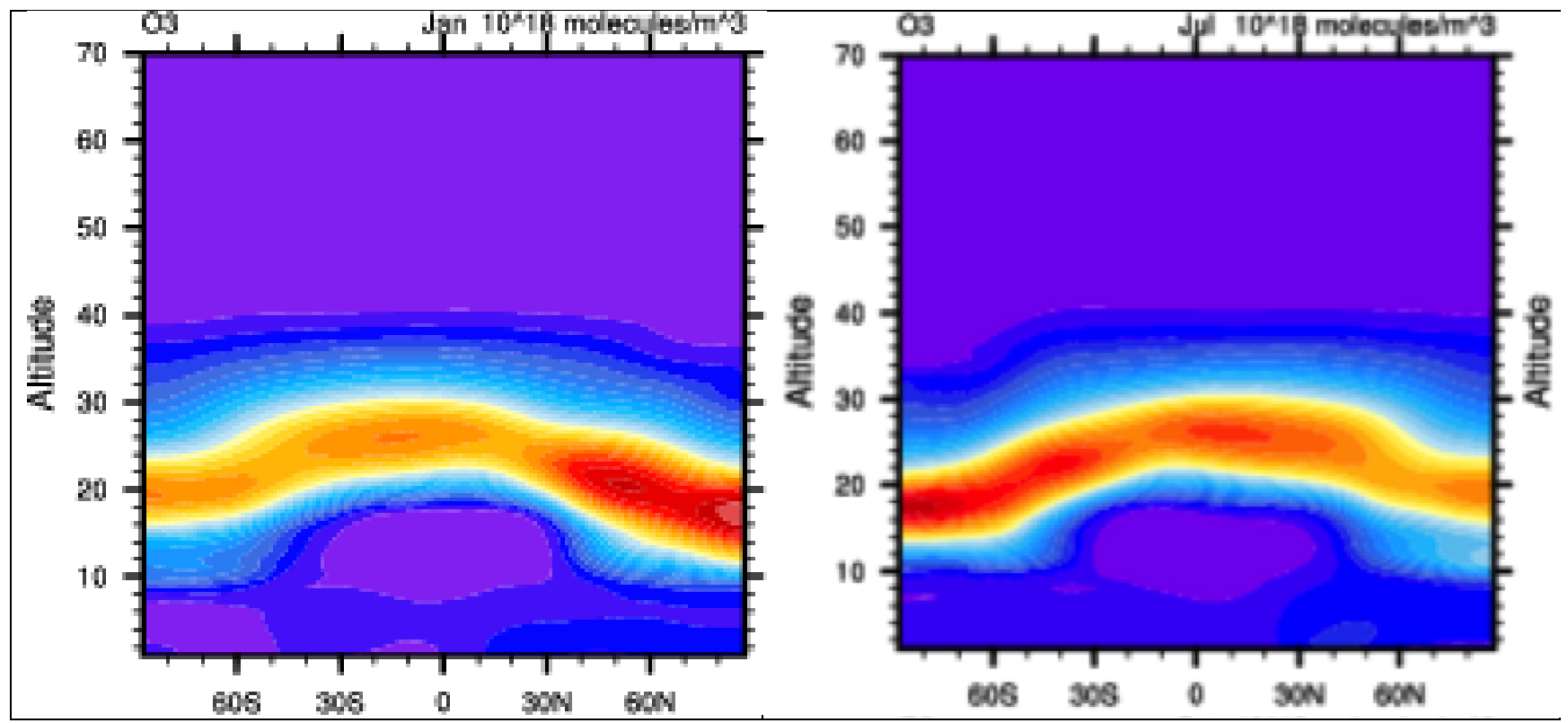

Figure 5: Seasonal variation in the ozone concentration with altitude. Credit: Retrieved from

https://climatedataguide.ucar.edu/climate-data/stratospheric-ozone-vertically-resolved-zonal-mean-based$\underline{\mathrm{bdbp}}$

\section{INSTRUMENT REQUIREMENTS}

\subsection{Platform Stability}

The telescope platform, suspended below the lifting balloon body, will suffer significant pendulum motion due to wind buffeting. Preliminary platform stability data for Project Loon has been made available to the project but remains confidential at this time. Active analysis is underway that indicates the level of active pointing stabilization required is achievable both in terms of update frequency and amplitude based on the current optical design. No inertial guidance package has been identified that would maintain sufficient pointing stability, hence the stabilization signal will be provided by a commercial star-tracker system, with a number of packages currently under investigation for performance suitability. Preliminary analysis indicates that a number of contemporary commercial compact, low-power, star-trackers available are capable of meeting the image quality budgeted for $\geq 80 \%$ platform stability data to hand. Some data loss due to uncorrectable deviations is deemed acceptable.

\subsection{Thermal stability}

At altitude, the telescope and electronics component will be subject to significant thermal and environmental variations. A robust, lightweight and athermal design will prove challenging. This will be addressed early in the Preliminary Design Study as it represents a major risk factor for the program viability. The availability of power to actively heat components, as well as opportunities to radiate away waste heat when required, are yet to be addressed.

\subsection{Detector selection}

The first phase of the GLUV mission will deploy a commercially available detector package in order to minimize development costs and time scales associated with this aspect of the program. A CCD sensor has been selected in the first instance, focusing on the near-ultraviolet $(250 \mathrm{~nm}<\lambda<400 \mathrm{~nm})$ due to limited available information on the true UV background and transmission at the accessible platform altitudes. Based on past experience with their excellent range of prepackaged systems, used extensively at ANU for laboratory systems, we have selected a Finger Lakes Instrumentation ProLine PL4240 UV package (based around the e2v CCD42-40-1-075 http://www.flicamera.com/proline/index.html). The large format, 2048 $\times 2048$ pixel, back illuminated detector has a high UV quantum efficiency (Figure 6) and modest readout noise and dark current for its operating temperature of $-40^{\circ} \mathrm{C}$. The $13.5 \mu \mathrm{m}$ pixels can be accommodated into practical optical designs. 


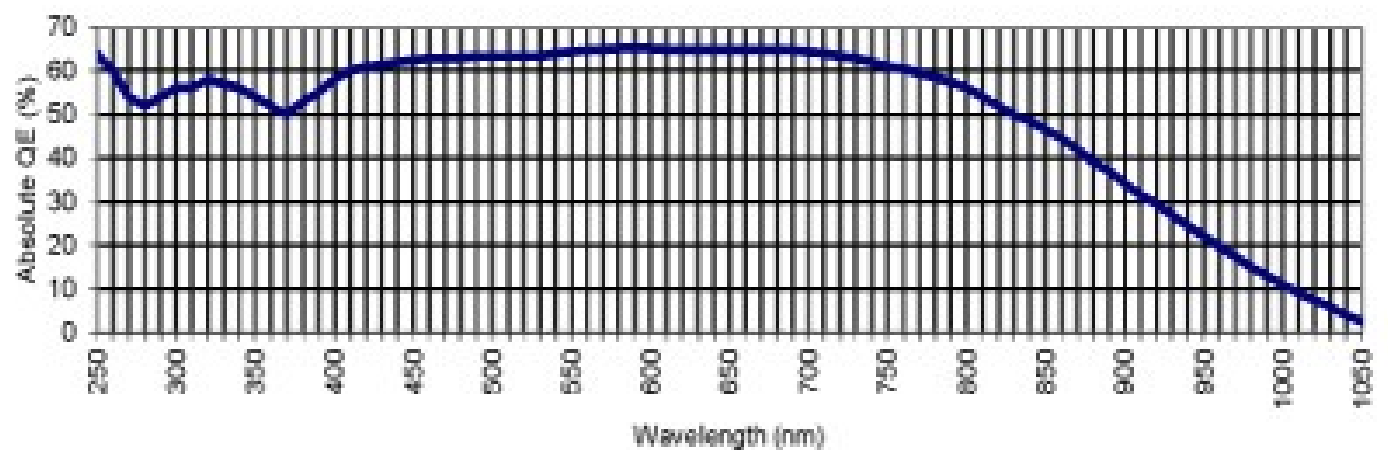

Figure 6: The Finger Lakes Instrument ProLine 4240 UV detector packages uses the e2v CCD42-40-1-075 CCD, providing excellent near-UV performance for a Silicon based detector.

\section{OPTICAL DESIGN}

A preliminary optical design has been prepared meeting the science requirements outline above. The design was significantly informed by review of the $\operatorname{CSTAR}^{[8]}$ telescope concept as GLUV shares a number of key design goal with the elegant CSTAR. The catadioptric design is shown in Figure 7. The early stage design meets the key specifications although we note that baffling will prove challenging if significant vignetting is to be avoided.

At the proposed operating in wavelengths transmissive elements are proposed to be composed of Suprasil-312 as the glass provide acceptable transmission properties without exhibiting unwanted fluorescence effects. The primary mirror diameter of $200 \mathrm{~mm}$ has a significant central obstruction reducing the effective collecting area to $55 \%$ the full aperture. The physical design length is $560 \mathrm{~mm}$, although this does not include forward baffling, which is expected to be significant. All elements are spherical in the baseline design with the expectation of the elliptical primary.

The effective focal length of $600 \mathrm{~mm}$ ( $\mathrm{f} \# 3$ ) leads to a plate scale of $343.8 \mathrm{arcsec} \mathrm{mm}^{-1}$, or $4.64 \mathrm{arcsec} \mathrm{pixel}^{-1}$ for the 13.5 $\mu \mathrm{m}$ pixels of the PL4240 detector package. The radial field is $\pm 1.86^{\circ}$, matched to the $2048 \times 2048$ pixel detector diameter, giving a nominal field of view at the detector of $\approx 7 \mathrm{deg}^{2}$. 

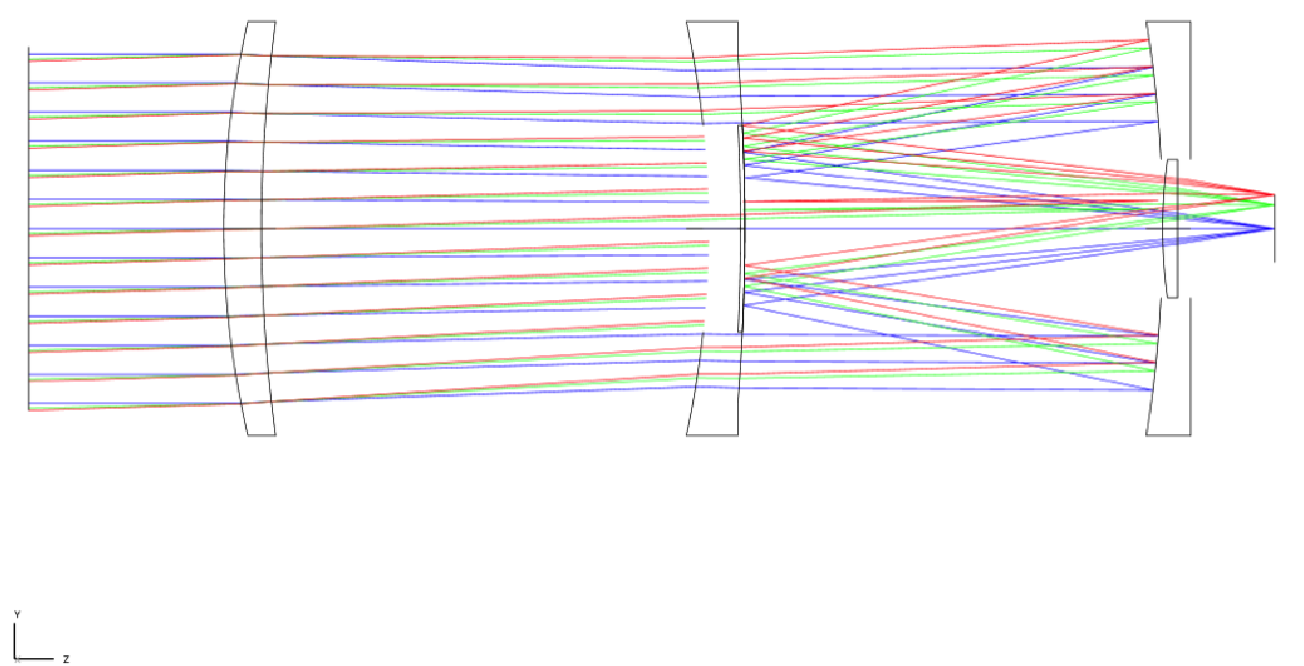

Figure 7: Our five element catadioptric design was informed by reference to the CSTAR $^{[8]}$ telescope concept.

\section{SUMMARY}

The GLUV project capitalise on the interesting opportunities made available by the proliferation of high-altitude longduration balloon missions coming on-line towards the end of the decade. It provides a route to access key observations not currently available for a number if frontier astronomical research programs. The GLUV Preliminary Design Study will define the first flight model for what is expected to be a suite of similar facilities providing a range of capabilities.

The program will be funded through an application to the Australia Research Councils Linkage Project program in October 2016. The three-year funding program will provide $50 \%$ of the necessary funding, with the reminder provided by a consortium of external parties including the Australia National University. Funding is expected to commence in the first quarter of 2017. The programs science drivers and requirements are currently under active development to ensure a formal Preliminary Design Study can be undertaken in 2017. Fabrication, integration and testing is plan for 2018 with the first test flights expected to be undertaken in 2018/19.

\section{REFERENCES}

1. Bloom, J. S., Kasen, D., Shen, K. J., Nugent, P. E., Butler, N. R., Graham, M. L., Howell, D. A., Kolb, U., Holmes, S. Haswell, C. A., Burwitz, V., Rodriguez, J., Sullivan, M., "A Compact Degenerate Primary-star Progenitor of SN 2011fe," The Astrophysical Journal, 744, 17 (2012)

2. Olling, R. P., Mushotzky, R., Shaya, E. J., Rest, A., Garnavich, P. M., Tucker, B. E., Kasen, D., Margheim, S., Filippenko, A. V., "No signature of ejecta interaction with a stellar companion in three type Ia supernovae," Nature, 521, $332,(2015)$

3. Kasen, D., Ramirez-Ruiz, E., "Optical Transients from the Unbound Debris of Tidal Disruption,” The Astrophysical Journal, 714, 155, (2010)

4. Hayden, B. T., Garnavich, P.M., Kasen, D., Dilday, B.; Frieman, J. A., Jha, S. W., Lampeitl, H., Nichol, R. C., Sako, M., Schneider, D. P., Smith, M., Sollerman, J., Wheeler, J. C., "Single or Double Degenerate Progenitors? Searching for Shock Emission in the SDSS-II Type Ia Supernovae," The Astrophysical Journal, 722, 1691-1698 (2010) 
5. Fressin, F., Torres, G., Charbonneau, D., Bryson, S. T., Christiansen, J., Dressing, C. D., Jenkins, J. M., Walkowicz, L. M., Batalah, N. M., "The False Positive Rate of Kepler and the Occurrence of Planets", The Astrophysical Journal, 766, $81(2013)$

6. Li, S.-Z, Yu, Y.-W., "Shock breakout driven by the remnant of a neutron star binary merger: An X-ray precursor of mergernova emission", The Astrophysical Journal, 819, 120 (2016)

7. McCormick, M. P., Zawodny, J. M., Veiga, R. E., Larsen, J. C., Wang, P. H., “An overview af SAGE I and II ozone measurements," Planetary and Space Science 37,1567-1586 (1989)

8. Yuan, X., Cui, X., Liu, G., Zhai, F., Gong, X., Zhang, R., Xia, L., Hu, J., Lawrence, J. S., Yan, J., Storey, J. W. V., Wang, L. Feng, L., Ashley, M. C. B., Zhou, X., Jiang, Z., Zhu, Z., "Chinese Small Telescope ARray (CSTAR) for Antarctic Dome A," Proc. SPIE, 7012, (2008) 